\title{
INTERVENÇÕES FARMACÊUTICAS CONTRIBUINDO PARA O USO RACIONAL DE MEDICAMENTOS EM TERAPIA INTENSIVA
}

Tamires Mietto Bressan (IC), Aline Rodrigues Teotonio (PG), Patricia Moriel (PQ), Profa. Dra. Priscila Gava Mazzola (PQ)

\section{Resumo}

Sabendo da variedade de medicamentos utilizados por pacientes numa Unidade de Terapia Intensiva (UTI), o cuidado com os riscos relacionados à farmacoterapia utilizada deve ser redobrado. Nesse contexto, este trabalho tem por objetivo avaliar o impacto das intervenções farmacêuticas no uso de medicamentos em UTI, realizado por meio de registro, quantificação e classificação de tais intervenções. Esse estudo contribui para salientar a importância das intervenções farmacêuticas e desenvolver essa ferramenta no contexto das UTIs da rede pública de saúde.

Palavras Chave: intervenção farmacêutica, UTI, farmácia clínica.

\section{Introdução}

Sabe-se que a Unidade de Terapia Intensiva (UTI) de um hospital é o local onde se concentram os pacientes que estão em uma situação de saúde emergente e que, portanto, precisam de maior atenção e cuidado. ${ }^{1}$ Dessa forma, o farmacêutico clínico intensivista tem um papel considerável dentro da UTI, já que possibilita o controle de interações medicamentosas e contribui para uma real melhoria na segurança e na qualidade do atendimento ao paciente. ${ }^{2,3}$ Os objetivos do estudo foram avaliar, quantificar e descrever 0 perfil das intervenções farmacêuticas e sua contribuição na segurança do uso de medicamentos em unidade de terapia intensiva UTI da rede pública.

\section{Resultados e Discussão}

A coleta deste projeto de iniciação científica teve duração de quatro meses. A aluna de iniciação científica registrou, classificou e quantificou as intervenções farmacêuticas. A coleta de prescrições ocorreu entre fevereiro e maio de 2015, contando com 152 pacientes, todos acima de 18 anos, admitidos na UTI geral adulto do Hospital de Clínicas da Unicamp. Foram analisadas 657 prescrições. Foram realizadas no período de estudo 315 Intervenções Farmacêuticas (IF). Foram feitas 292 intervenções relacionadas a interações medicamentosas potenciais (IMP). Foram feitas ainda 13 intervenções para correção de erros de prescrição, 10 para ajuste de dose maior que a indicada e 3 para correção de unidade utilizada na prescrição. Foram feitas ainda 10 intervenções de pesquisa, solicitadas pela equipe intensivista para o esclarecimento de dúvidas referentes aos medicamentos ou solicitação de informações para atualização da equipe. A aceitação das intervenções pela equipe foi total, $100 \%$ das intervenções realizadas foram aceitas. A média de intervenções por paciente foi de $4,1 \mathrm{com}$ desvio padrão de 3,9. Essa média é explicada pelo fato de que as intervenções de informação não são atribuídas a pacientes específicos, sendo geralmente contabilizadas apenas no total de intervenções. Como a maior parte das intervenções são referentes às IMP, essa variação é vista tanto para as IMP quanto para as IF.

\section{Conclusões}

O elevado número de intervenções encontrado e a elevada aceitação da equipe demonstra a necessidade de discussão sobre o manejo da farmacoterapia utilizada em terapia intensiva, principalmente com relação a IMPs, além de reforçar o caráter multiprofissional da equipe de terapia intensiva.

\section{Agradecimentos}

Agradeço à equipe de pesquisa por me proporcionar a oportunidade de enriquecer meus conhecimentos com esse projeto e valorizar o meu trabalho.

\footnotetext{
${ }^{1}$ YUNES, L. P.; COELHO, T. A.; ALMEIDA, S. M. Principais interações medicamentosas em pacientes da UTI-adulto de um hospital privado de Minas Gerais. R. Bras. Farm. Hosp. Serv. Saúde,São Paulo, v. 2, n. 3, p. 23-26, set./dez. 2011.

${ }^{2}$ RUDIS, M.I.; BRANDL, K.M.. Position paper on critical care pharmacy services. Crit Care Med,[S. 1.], v. 28, n. 11, p. 37463750, nov. 2000.

3MAZZOLA, P.G.; RODRIGUES, A.T.; DA CRUZ, A. A.; DE MARIALVA, M.; GRANJA, S.; BATTAGLINI, S. C. M.; FALCÃO, A. L. E.; MORIEL,P. "Perfil e manejo de interações medicamentosas potenciais teóricas em prescrições de UTI. R. Bras. Farm. Hosp. Serv. Saúde, São Paulo, v. 2, n. 2, p. 15-19, mai./ago. 2011.

${ }^{4}$ OVERHAGE, J.M.; LUKES, A. Practical, reliable, comprehensive method for characterizing pharmacists clinical activities. Am. J. Health Syst. Pharm., [S. 1.], v.56, n. 23, p. 2444-2450, dez. 1999.
} 